\title{
Processing and analysis of transient pressure measurements from permanent down-hole gauges
}

\author{
Wang Fei* \\ School of Petroleum Engineering, China University of Petroleum, Beijing 102249, China \\ (C) China University of Petroleum (Beijing) and Springer-Verlag Berlin Heidelberg 2012
}

\begin{abstract}
With permanent down-hole gauges (PDGs) widely installed in oilfields around the world in recent years, a continuous stream of transient pressure data in real time is now available, which motivates a new round of research interests in further developing pressure transient processing and analysis techniques. Transient pressure measurements from PDG are characterized by long term and high volume data. These data are recorded under unconstrained circumstances, so effects due to noise, rate fluctuation and interference from other wells cannot be avoided. These effects make the measured pressure trends decline or rise and then obscure or distort the actual flow behavior, which makes subsequent analysis difficult. In this paper, the problems encountered in analysis of PDG transient pressure are investigated. A newly developed workflow for processing and analyzing PDG transient pressure data is proposed. Numerical well testing synthetic studies are performed to demonstrate these procedures. The results prove that this new technique works well and the potential for practical application looks very promising.
\end{abstract}

Key words: Permanent down-hole gauge, transient pressure, data processing, deconvolution technique, numerical well testing

\section{Introduction}

A permanent down-hole gauge (PDG) is a pressure and temperature real time monitoring system installed at the bottom hole near the reservoir. Its main objective is to help the reservoir management and the production by continuously measuring pressure and temperature in the reservoir (Chorneyko, 2006). PDGs have been widely installed in oilfields around the world in recent years.

Transient pressure data from permanent down-hole gauges are long term and high volume, as shown in Fig. 1. Usually the whole sequence of PDG pressure comprises hundreds of pressure drawdown (PDD) and pressure buildup (PBU) flow periods. So it has the potential to provide more information about a reservoir than those from relatively short test durations. However, the current pressure transient analysis (PTA) techniques are mainly based on the analysis of individual flow periods in isolation, namely PDD or PBU period in the test (Du, 2007). Therefore, it becomes a challenge to analyze PDG transient pressure with the existing PTA techniques.

Moreover, there are several issues related to this type of long-term PDG data, such as the data are inherently noisy because they are obtained under uncontrolled conditions

*Corresponding author. email: wangfei@cup.edu.cn Received August 8, 2011

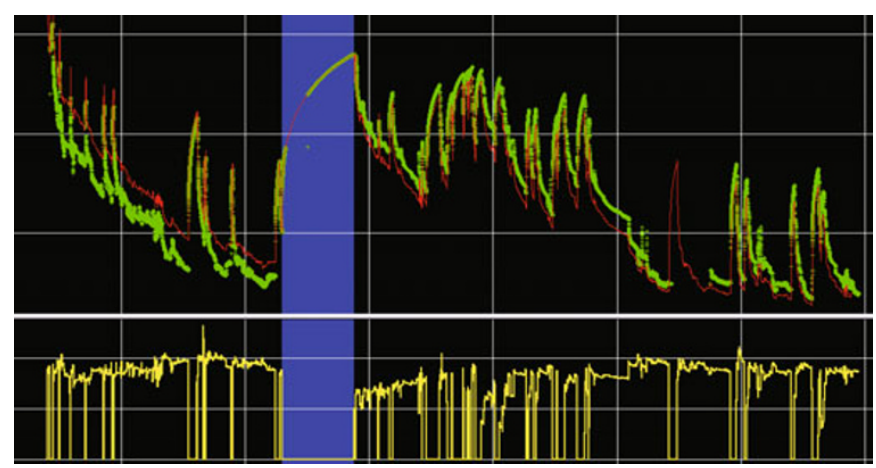

Fig. 1 PDG transient pressure data

(Athinichanagorn, 1999). Besides, the measured long-term pressures, which combined test and production data, are both achieved under variable conditions. In practice it is impossible to keep a constant flow condition for obtaining transient rate or transient pressure, so the practical data set is either variable-rate transient pressure or variable-pressure transient rate. While current theoretical methods for rate transient analysis (RTA) and pressure transient analysis (PTA) in well testing are based on constant rate or pressure solutions, which means before the transient analysis, the transient data (pressure or rate) need to be normalized to that due to either a constant-rate or constant-pressure form (Lee and Spivey, 1998; Mohaghegh et al, 2005; Gaskari et al, 2006). 
In addition to the noise problem and variable-rate superposition effect, multi-well interference is another common issue in practice for PDG transient pressure measurements (Britt et al, 1991; Erwin et al, 2002). Interference between wells affects the measured pressure trends and obscures or distorts the measured flow behavior, which makes subsequent analysis difficult (Leaver et al, 1988; Onur et al, 1991; Hallford and Hegeman, 1995; Marhaendrajana et al, 2003; Bischoff and Bejaoui, 2005).

For the multi-rate superposition effect, which is inevitable in PDG pressure measurements, there have been several classical rate normalization methods. The purpose is to make sure normalized data, namely rate-normalized pressure, fit the traditional pressure transient theory. Methods published in the literature on this subject area include various types of superposition and normalization methods (Palacio and Blasingame, 1993; Agarwal et al, 1999). Deconvolution (Roumboutsos and Stewart, 1988) is one of these rate normalization methods because it can transfer the variable rate pressure data into an equivalent pressure response due to the constant rate profile. This equivalent constant rate pressure response can be used for reservoir model identification. Deconvolution techniques have been applied to long-term PDG data in recent years. Hollaender et al (2001) and Schroeter et al (2002), presented a deconvolution technique by considering deconvolution as a nonlinear total least squares problem. Levitan et al (2004) also produced a more practical deconvolution algorithm by utilizing an unconstrained, nonlinear weighted least-squares objective function involving the sum of three mismatch terms for pressure, rate and curvature. Pimonov et al (2009) presented a pressure-pressure deconvolution algorithm, through which the flow rate is eliminated from the convolution formulation, so it can be used to analyze multipoint pressure transient data such as interference and MDT tests. In processing multiwell interference effects, traditional methodology is from multiple well tests (Kamal, 1983). The pressure response is measured in an observation well some distance away from the active well, which may be a producing or an injection well. Through the analysis of the observation well, average reservoir properties in the area separating the wells are determined. However, a drawback of multiple well tests is that it requires one or more potentially productive wells to be shut-in (Bourdet, 2002). Multi-well interference is very common in PDG data. It makes the measured pressure trends decline or rise and then obscures or distorts the underlying flow behavior, which makes the subsequent analysis difficult, i.e. the construction of the incorrect semi-log straight line or the incorrect radial flow regime on a pressure derivative log$\log$ plot. Zheng and Wang $(2009 ; 2010)$ presented a two-well deconvolution method for well interference extraction and analysis.

All the stated issues above, i.e. long-term, noise problem, variable-rate superposition and multi-well interference effects in PDG transient pressure data make the straightforward interpretation unavailable due to the limitation of the current PTA and RTA theory. Therefore, this paper attempts to understand the problems in processing and analyzing PDG transient pressure data and to develop a more practical method to solve them.

\section{Workflow for processing and analyzing PDG data}

Our workflow for processing and analyzing PDG data, shown in Fig. 2, includes four steps: collecting data, processing data, analyzing data and updating the reservoir model (Wang, 2010).

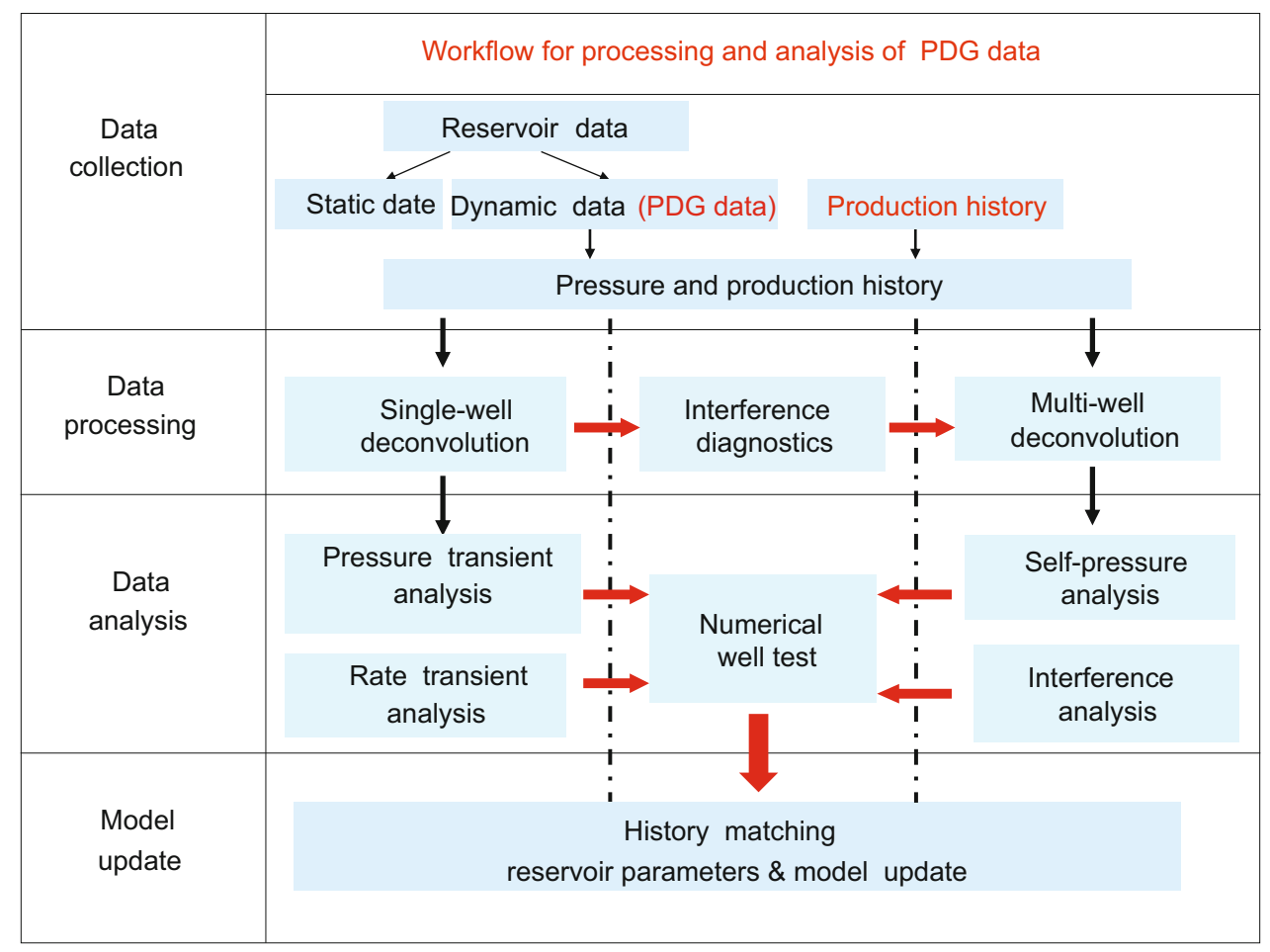

Fig. 2 Workflow for processing and analyzing PDG data 
In the data collection step, we focus on the dynamic data, especially PDG pressure and production data. Then we process and analyze the PDG data in two different conditions: single-well deconvolution method could solve the pressure/ rate variations and use nonlinear least square optimization to solve the noise problem. A multi-well deconvolution method could additionally solve the well interference problem. After the data processing, the deconvolution-based rate and pressure transient analysis methods could be used. Reservoir parameters and production forecasting could be estimated. Finally after numerical well testing we update the reservoir model and integrate all the results by future history match.

\section{Deconvolution transformand deconvolution-based data analysis}

\subsection{The procedure of the single-well deconvolution method to process and analyze PDG data}

The procedure of the single-well deconvolution method to process and analyze PDG data includes the following two steps:

1) Data processing

- Pressure-rate deconvolution transform

- Rate-pressure deconvolution transform

2) Data analysis

- Deconvolution-based pressure transient analysis

- Deconvolution-based rate transient analysis

The procedures, as shown in Fig. 3, include first, the processing of the variable pressure and rate data with two single-well deconvolution algorithms respectively. Once the deconvolved pressure/rate, where deconvolved pressure is for pressure transient analysis and deconvolved rate is for rate transient analysis, is obtained, these deconvolutionbased analysis methods can be used for reservoir system identification and parameter estimation at the same time. As the theory for both methods are the same, the results from transient-pressure analysis and transient-rate analysis can support each other to ensure the result is more reliable.

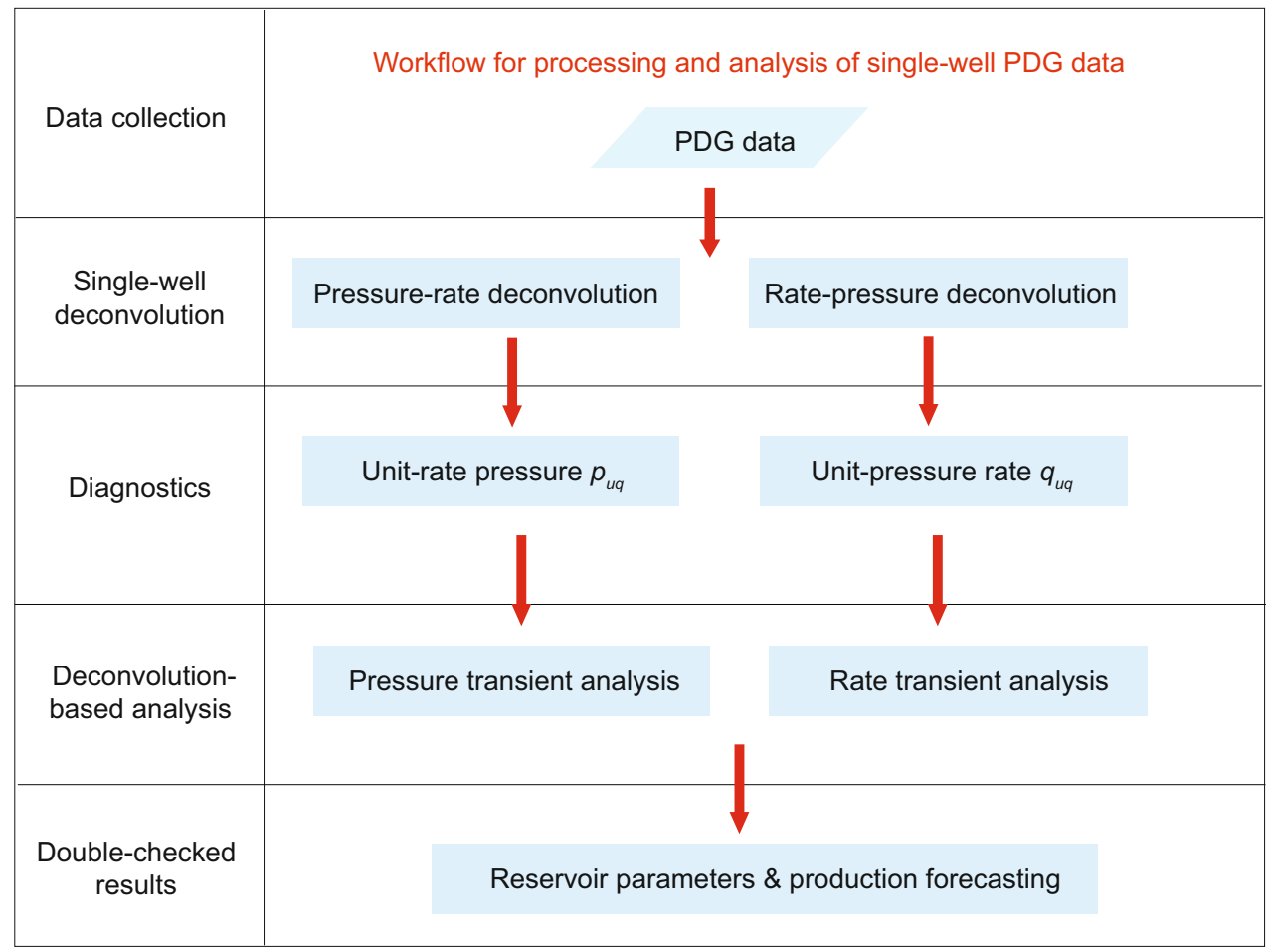

Fig. 3 Diagram of processing and analysis of single-well PDG data

\subsection{The procedure of multi-well deconvolution method to process and analyze PDG data}

The procedure of multi-well deconvolution method to process and analyze PDG data includes following three steps:

1) Well interference extraction

2) Near-well data analysis

3) Inter-well data analysis

The procedures are shown in Fig. 4. Firstly, we use the multi-well deconvolution transform algorithm to extract the well interference from the total pressure response and obtain the decomposed well self-pressure response $p_{\text {uii }}$ and the well interference response $p_{u i j}$. Finally deconvolution-based self- pressure analysis can be used to obtain near-well properties. While, interference analysis of the extracted $p_{u i j}$ can be used to obtain inter-well properties.

A synthetic study of numerical well testing is performed to demonstrate the above mentioned procedures for both single and multiple wells, as shown in Fig. 5.

Fig. 5(a) shows the whole flowing history for the well. This data has been synthetically generated from a fractured well model. The data is close to a PDG field example. The longest pressure drawdown period of this sequence on log-log plot is shown in Fig. 5(b), in which just one linear flow, i.e. fracture linear flow, can be investigated. Apparently, the analysis of individual flow periods, i.e. analyzing the longest drawdown 


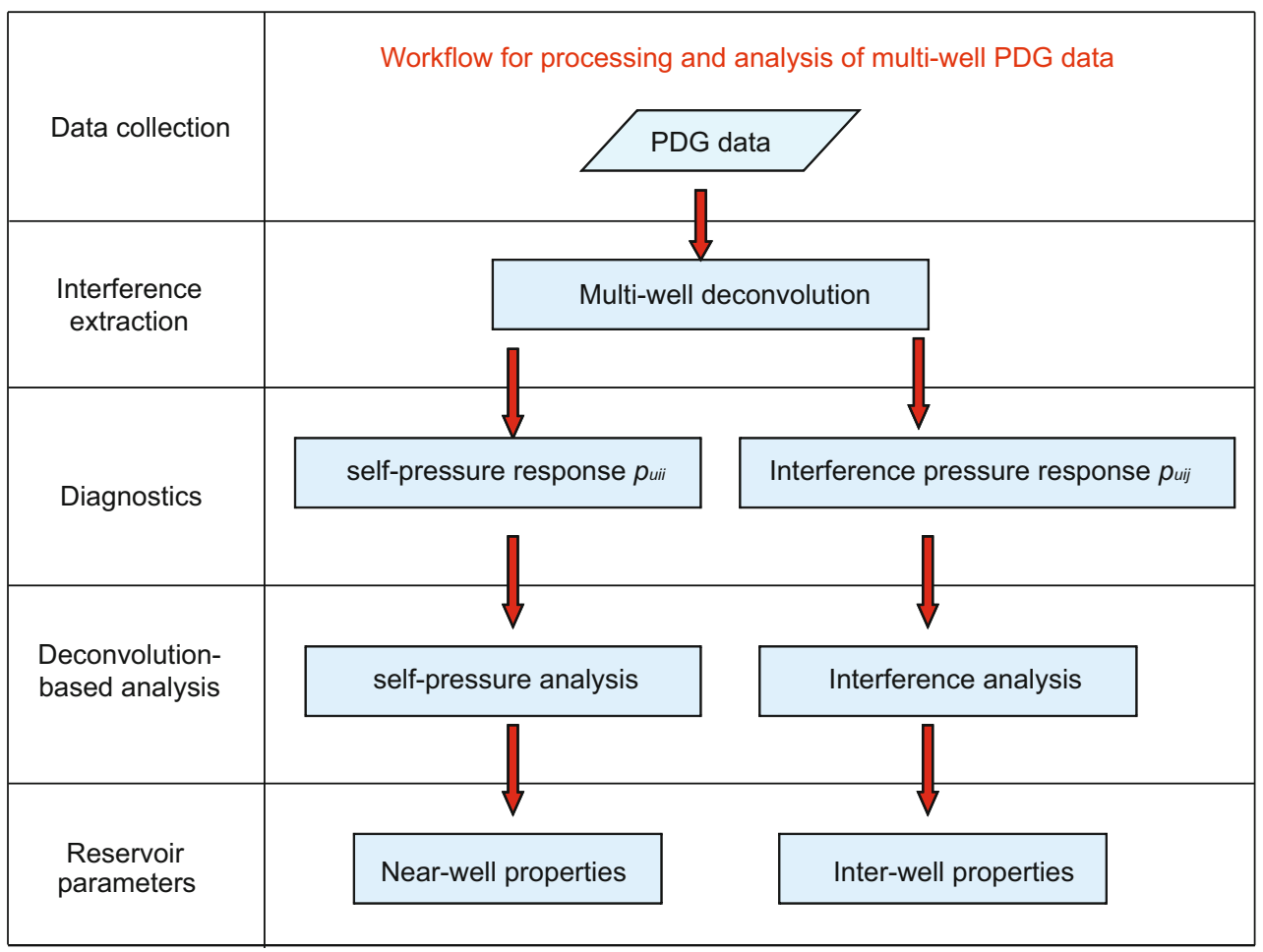

Fig. 4 Diagram of processing and analysis of multi-well PDG data

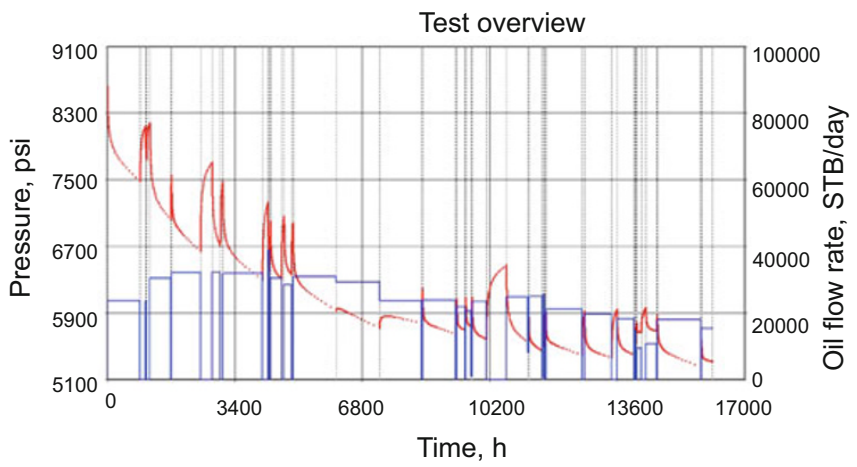

(a)

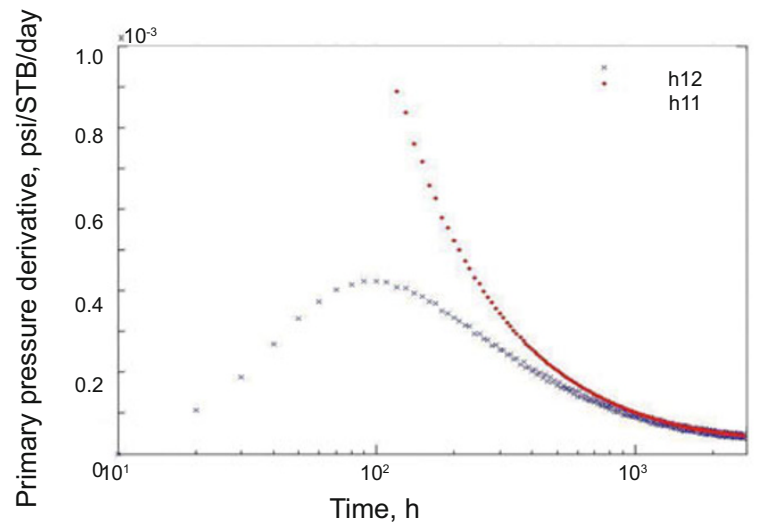

(c)

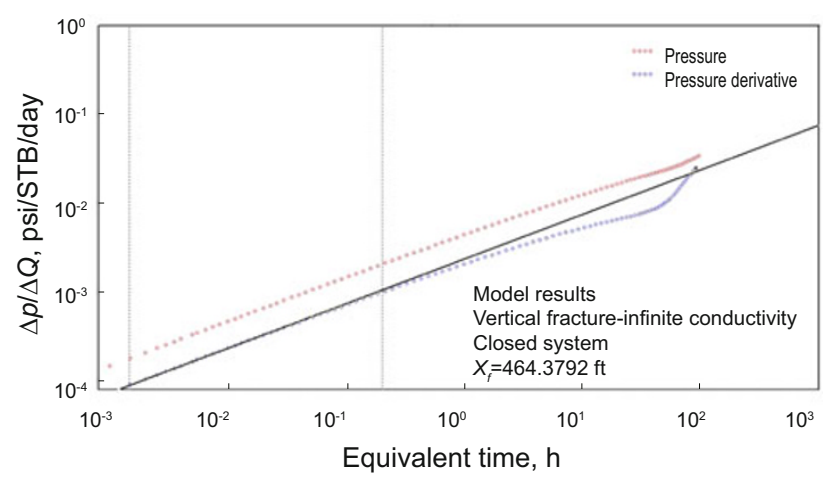

(b)

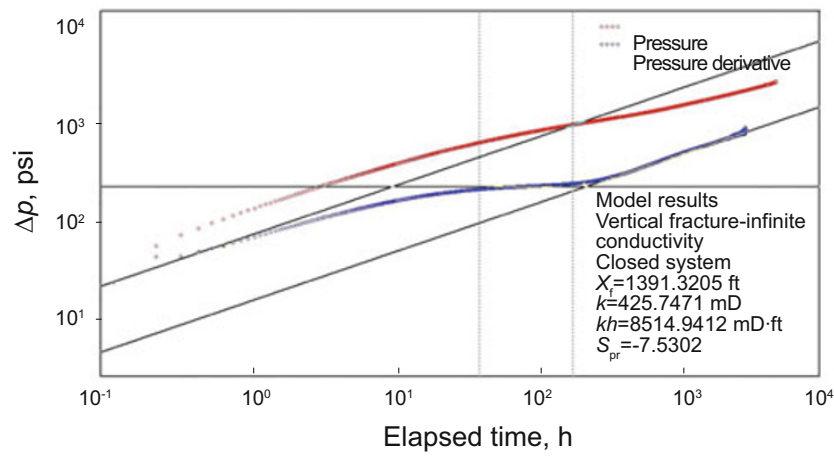

(d)

Fig. 5 Diagram of a numerical well testing synthetic case

above, cannot determine the reservoir boundary type.

The multi-well deconvolution algorithm is implemented on the pressure and rate data of the well. The interference pressure response $p_{u 12}$ is extracted with our developed algorithm. Besides, the self-pressure response $p_{u 11}$ of Well 1 can be obtained simultaneously. Their primary derivatives are put on a linear plot for comparison, shown in Fig. 5(c).

Fig. 5(d) shows the deconvolved self-pressure and 
its derivative on a log-log plot. It is clearly the second linear flow, i.e. reservoir linear flow, since there is no flat slope line followed by the half slope line. So the reservoir boundary type can be identified as parallel faults. Thereafter deconvolution-based pressure transient analysis can be used to calculate the reservoir parameters.

\section{Application of deconvolution techniques to PDG data}

For applying deconvolution techniques to PDG data in multi-well reservoir conditions, a three-step methodology is proposed, which includes well interference diagnostic, extraction and analysis.

The procedures, shown in Fig. 6, include firstly, the diagnosis of the reservoir system response for the nonlinearity. As soon as the reason causing this nonlinearity due to well interference is found, the following step is to extract the interference from the total pressure response. Then, the analysis of the decomposed data can be made using the available traditional well testing methods.

For interference diagnosis, a single-well deconvolution can transfer the transient pressure as a result of variable rate into an equivalent unit-rate transient pressure. It also can convert a series of transient pressures, due to variable or step rate history into an equivalent unit-pressure transient rate. Once such responses (unit-rate pressure and unit-pressure rate) are generated, they can be used to identify the reservoir model and diagnose the occurrence of interference.

For interference extraction, multi-well deconvolution can be used to separate interference information from long-term real-time PDG data and then generate equivalent constantrate pressure and constant-pressure rate data. The processed data can be used for regular analysis.

For interference analysis, transient pressure analysis and transient rate analysis are implemented on the deconvolved pressure and rate data. The results of two deconvolutionbased analyses show a good match, which proves that deconvolution is a reliable technique multi-well reservoir conditions.

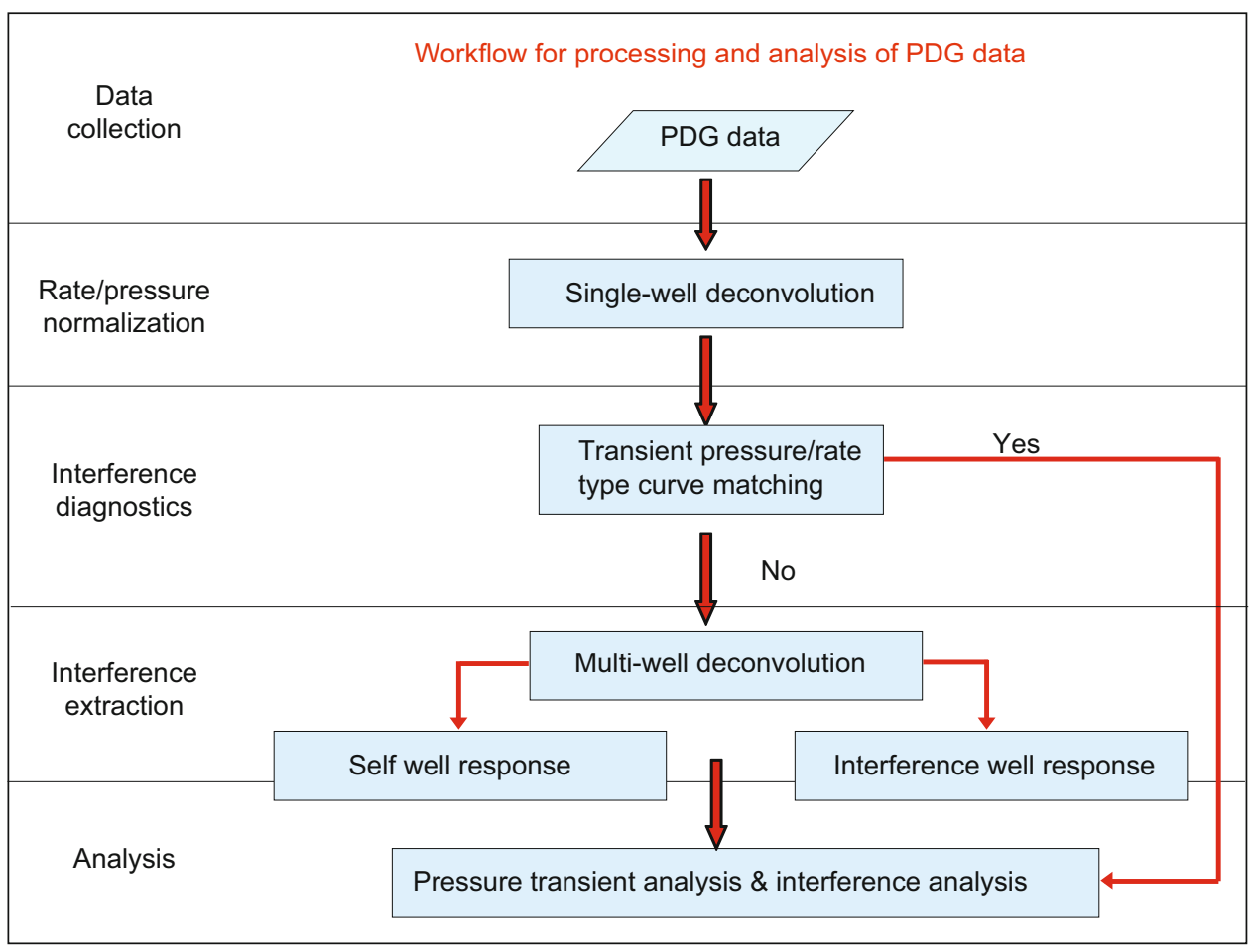

Fig. 6 Diagram of processing and analysis of PDG data

\section{Discussion}

Deconvolution as a new tool to process PDG transient data, makes available more long-term large-volume transient pressure interpretation and therefore more additional reservoir information can be obtained. However, there are some limitations so this technique cannot be routinely utilized in practice.

The basic assumption of all deconvolution techniques is the consistency of measured pressure and rate data with the linear Duhamel model, which is based on the principle of superposition. The linearity of the system suggests that initial equilibrium state must be satisfied during the deconvolution procedure. That means the interpretation model cannot change during the data processing. Another requirement for linearity of the system is the single-phase flow, which means that the down-hole pressure for deconvolution must be higher than that at the bubble point. Once multi-phase flow exists, deconvolution cannot work. 


\section{Conclusions}

It has been noted that the current RTA and PTA methods are not satisfactory with PDG transient pressure data. Preanalysis data processing is required so that the problems of long-term, noise, variable-rate superposition and multiwell interference effects in PDG transient pressure data can be resolved and traditional analysis methods can be fully utilized.

This paper presented a new deconvolution transform method, which is suitable for processing and analyzing transient pressure data from permanent down-hole gauges. After processing, the pressure/rate variations can be solved and the inter-well interference can be extracted. The entire history of PDG transient pressure can be analyzed. Therefore additional reservoir diagnostics and more distant reservoir features, such as reservoir boundaries can be obtained.

\section{Acknowledgements}

The author would like to thank Science Foundation of China University of Petroleum, Beijing (No.YJRC-2011-02) for the financial support during this research.

\section{References}

Agarwal R G, Gardner D C, Kleinsteiber S W and Fussell D D. Analyzing well production data using combined-type-curve and decline-curve analysis concepts. SPE Reservoir Evaluation and Engineering. 1999. 10: 478-486 (SPE 49222)

Athinichanagorn S. Development of an Interpretation Methodology for Long-term Data from Permanent Downhole Gauges. Ph.D Thesis. Stanford University, Department of Petroleum Engineering. 1999

Bischoff R and Bejaoui R. Integrated modeling of the mature Ashtart Field, Tunisia. SPE Europec/EAGE Annual Conference, 13-16 June 2005, Madrid, Spain (SPE 94007)

Bourdet D. Well Test Analysis: The Use of Advanced Interpretation Model. Handbook of Petroleum Exploration and Production. 2002. 273-301

Britt L K, Jones J R, Pardinl R E and Plum G L. Reservoir description by interference testing of the Clayton Field. Journal of Petroleum Technology. 1991. 43(5): 524-527, 575-577 (SPE 19846-PA)

Chorneyko D M. Real-time reservoir surveillance utilizing permanent downhole pressures: An operator's experience. SPE Annual Technical Conference and Exhibition, 24-27 September 2006, San Antonio, Texas, USA (SPE 103213)

Du K F. Use of advanced pressure transient analysis techniques to improving for drainage area calculations and reservoir characterizations: Field case studies. Offshore Europe, 4-7 September 2007, Aberdeen, UK (SPE 109053)

Erwin M D, Sander L A and Redman R S. Multiwell interference test in the Colville River Field, Alaska. SPE Annual Technical Conference and Exhibition, 29 September-2 October 2002, San Antonio, Texas (SPE 77453)

Gaskari R, Mohaghegh S D and Jalali J. An integrated technique for production data analysis with application to mature fields. SPE Gas Technology Symposium, 15-17 May 2006, Calgary, Alberta, Canada (SPE 100562)
Hallford D L and Hegeman P S. A field application of multiplewell testing for reservoir characterization. SPE Annual Technical Conference and Exhibition, 22-25 October 1995, Dallas, Texas (SPE 30575)

Hollaender F, Schoreter T and Gringarten A C. Deconvolution of well test data as a nonlinear total least squares problem. SPE Annual Technical Conference and Exhibition, October 2001, New Orleans, Louisiana (SPE 71574)

Kamal M M. Interference and pulse testing: A review. Journal of Petroleum Technology. 1983. 12: 2257-2270

Leaver J D, Grader A and Ramey H J. Multiple-well interference testing in the Ohaaki Georhermal Field. SPE Formation Evaluation. 1988. 3(2): 429-437 (SPE 15122)

Lee W J and Spivey J P. Numerical and analytical well test analysis: A case history. SPE Annual Technical Conference and Exhibition, 2730 September 1998, New Orleans, Louisiana (SPE 50946)

Levitan M M, Crawford G E and Hardwick A. Practical considerations of pressure-rate deconvolution of well test. SPE Annual Technical Conference and Exhibition, September 2004, Houston, Texas (SPE 90680)

Marhaendrajana T, Ariadji T and Permadi A K. Performance prediction of a well under multiphase flow conditions. SPE Asia Pacific Oil and Gas Conference and Exhibition, 9-11 September 2003, Jakarta, Indonesia (SPE 80534)

Mohaghegh S D, Gaskari R and Jalali J. A new method for production data analysis to identify new opportunities in mature fields: Methodology and application. SPE Eastern Regional Meeting, 1416 September 2005, Morgantown, West Virginia (SPE 98010)

Onur M, Serra K V and Reynolds A C. Analysis of pressure-buildup data from a well in a multiwell system. SPE Formation Evaluation. 1991. 6(1): 101-110 (SPE 18123)

Palacio J C and Blasingame T A. Decline-curve analysis using type curves: Analysis of gas well production data. Joint Rocky Mountain Regional and Low Permeability Reservoirs Symposium, 26-28 April 1993, Denver, CO (SPE 25909)

Pimonov E, Ayan C, Onur M and Kuchuk EJ. New pressure/rate deconvolution algorithm to analyze wireline formation tester and well-test data. SPE Annual Technical Conference and Exhibition, 4-7 October 2009, New Orleans (SPE 123982)

Roumboutsos A and Stewart G. A direct deconvolution or convolution algorithm for well-test analysis. SPE Annual Technical Conference and Exhibition, October 1988, Houston, Texas (SPE 18157)

Schroeter T, Hollaender F and Gringarten A C. Analysis of well test data from downhole permanent downhole gauges by deconvolution. SPE Annual Technical Conference and Exhibition, 29 September-2 October 2002, San Antonio, Texas (SPE 77688)

Wang F. Processing and Analysis of Transient Pressure from Permanent Down-hole Gauges. Ph.D Thesis. Heriot-Watt University, Institute of Petroleum Engineering. 2010

Zheng S Y and Wang F. Extraction of interference from long-term transient pressure using multi-well deconvolution algorithm for well test analysis. CPS/SPE International Oil \& Gas Conference and Exhibition, 8-10 June 2010, Beijing, China (SPE131294)

Zheng S Y and Wang F. Multi-well deconvolution algorithm for the diagnostic, analysis of transient pressure with interference from permanent down-hole gauges. SPE Europec/EAGE Annual Conference and Exhibition, Amsterdam, 8-11 June 2009, Netherland (SPE121949)

(Edited by Sun Yanhua) 